\title{
Uso de la clasificación de Robson en un Hospital Comarcal de España para reducir la tasa de cesáreas
}

\author{
Rosario Aguilar Redondo ${ }^{1}$, Gador Manrique Fuentes ${ }^{1}$, Leonardo Mauro Aisa Denaroso ${ }^{1}$, \\ Luis Delgado Martínez ${ }^{1}$, Valois González Acosta ${ }^{1}$, Longinos Aceituno Velasco. ${ }^{1}$ \\ ${ }^{1}$ Servicio Andaluz de Salud. Área Gestión Sanitaria Norte Almería. Hospital La Inmaculada. Servicio de Obstetricia y \\ Ginecología. Huércal-Overa (Almería). Andalucía. España.
}

\section{RESUMEN}

Antecedentes: En las últimas décadas se ha producido un incremento extraordinario en la incidencia de cesáreas en todos los países desarrollados. La tasa de cesáreas en España registrada por el Ministerio de Sanidad es de 24,9\%. Objetivo: Analizar las cesáreas en el Hospital La Inmaculada, mediante la clasificación de Robson para comparar con otros hospitales, tanto nacional como internacionalmente y establecer las intervenciones posibles para reducir la tasa de cesáreas. Métodos: Se realiza una auditoría retrospectiva de cesáreas en función de la clasificación de diez grupos establecida por Robson desde el 1 enero de 2006 al 31 de diciembre de 2013. Resultados: Se han analizado 9337 partos y 1507 cesáreas con un $16,14 \%$. En la contribución al porcentaje de cesáreas en primer lugar con $25,2 \%$ corresponde a nulíparas con un feto único en presentación cefálica, de 37 semanas o más de embarazo. En segundo lugar las multíparas con al menos una cesárea previa, con un feto único en presentación cefálica, de 37 semanas o más de embarazo con $19,4 \%$. En este grupo se ha realizado un $42,2 \%$ de cesáreas. En tercer lugar nulíparas con un feto único en presentación cefálica, de 37 semanas o más de embarazo, que han iniciado el parto de forma espontánea con 17,4\%. Conclusiones: El aumento de cesáreas en las últimas décadas hace necesario la realización de auditorías mediante un sistema de clasificación, como Robson, para establecer los grupos en los que es posible disminuir el número de cesáreas.

\section{PALABRAS CLAVES: Cesáreas, clasificación de Robson, calidad de atención, auditoría médica}

\section{SUMMARY}

Introduction: In recent decades there has been a dramatic increase in cesarean section rate in all developed countries. The cesarean section rate in Spain, registered by the Ministry of Health was $24.9 \%$. Objective: To analyze cesarean section rate in Inmaculada Local Hospital, using Robson's classification to compare it with other hospitals, both at national and international level and establish potential interventions to reduce such rate. Methods: A cesarean section rate retrospective audit was performed according to Robson's classification, from 1st January 2006 to 31st December 2013. Results: 9337 deliveries and 1507 cesareans were analyzed on that period of time. The cesarean rate was $16.14 \%$. Nulliparous women with a singleton pregnancy in cephalic presentation at 37 weeks or more and undergo a labor induction before the onset of labor represent the first group of the cesareans with $25.2 \%$ of the total. In second place comes multiparous women with at least one previous cesarean section with a singleton pregnancy in cephalic presentation, 37 weeks or more, representing $19.4 \%$ of the total cesareans. This group had a $42.2 \%$ cesarean rate. In third place $(17.4 \%)$ we had nulliparous women with a singleton pregnancy in cephalic presentation, 37 weeks 
or more, who have started labor spontaneously. Conclusions: Increased cesarean section rate in recent years required audits using a classification system, like Robson's one, to establish the groups in which it is possible to reduce the number of cesareans

\section{KEY WORDS: Cesarean section, Robson's classification, quality of care; medical audit}

\section{INTRODUCCIÓN}

En las últimas décadas se ha producido un incremento extraordinario en la incidencia de cesáreas en todos los países desarrollados, incluyendo España $(1,2,3)$. La OMS incluye la tasa de cesáreas como un indicador de calidad de la atención materna y perinatal. En sus recomendaciones de 1985 refería como criterio de calidad una tasa de cesárea del 15\% (4).

La tasa de cesáreas en España y en otros países europeos se encuentra por encima de esos valores y viene incrementándose progresivamente. La tasa de cesáreas en España registrada por el Ministerio de Sanidad en 2011 es de 24,9\%.
Existen factores nuevos que podrían relacionarse con el incremento en la tasa de cesáreas, como el aumento de la edad de las madres, los partos múltiples, las solicitudes por parte de las mujeres y las demandas por sospecha de mala práctica $(4,5,6)$.

En 2001 hubo una propuesta de un sistema de clasificación para la monitorización de las cesáreas descrito por Robson y cols (Tabla I) que define 10 grupos sobre la base de 4 conceptos obstétricos: la categoría del embarazo (embarazo múltiple/único, presentación fetal), antecedentes obstétricos (nulíparas, multíparas con/sin cicatriz uterina), el curso del trabajo de parto y el parto (parto espontáneo/ parto inducido/cesárea antes del parto), y la edad gestacional al momento del parto (7).

\section{Tabla I \\ CLASIFICACIÓN DE ROBSON}

\begin{tabular}{|c|c|}
\hline Grupos & Descripción de la clasificación de diez grupos \\
\hline 1 & $\begin{array}{l}\text { Nulíparas con un feto único en presentación cefálica, de } 37 \text { semanas o más de embarazo, que } \\
\text { han iniciado el parto de forma espontánea. }\end{array}$ \\
\hline 2 & $\begin{array}{l}\text { Nulíparas con un feto único en presentación cefálica, de } 37 \text { semanas o más de embarazo, que } \\
\text { han sido sometidas a inducción o cesárea antes del inicio del parto. }\end{array}$ \\
\hline 3 & $\begin{array}{l}\text { Multíparas sin cesárea previa, con un feto en presentación cefálica, de } 37 \text { o más de embarazo, } \\
\text { que han iniciado el parto de forma espontánea. }\end{array}$ \\
\hline 4 & $\begin{array}{l}\text { Multíparas sin cesárea previa, con un feto en presentación cefálica, de } 37 \text { semanas o más de } \\
\text { embarazo, que han sido sometidas a inducción o cesárea antes del inicio del parto. }\end{array}$ \\
\hline 5 & $\begin{array}{l}\text { Multíparas con al menos una cesárea previa, con un feto único en presentación cefálica, de } 37 \\
\text { semanas o más de embarazo. }\end{array}$ \\
\hline 6 & Nulíparas con un feto único en presentación podálica. \\
\hline 7 & Multíparas con un feto único en presentación podálica, incluyendo aquellas con cesárea previa. \\
\hline 8 & Todas las mujeres con embarazo múltiple, incluyendo aquellas con cesárea previa. \\
\hline 9 & $\begin{array}{l}\text { Todas las mujeres con feto único en posición transversa u oblicua, incluyendo aquellas con } \\
\text { cesárea previa. }\end{array}$ \\
\hline 10 & $\begin{array}{l}\text { Todas las mujeres con un feto único en presentación cefálica de menos de } 37 \text { semanas de } \\
\text { embarazo, incluyendo aquellas con cesárea previa. }\end{array}$ \\
\hline
\end{tabular}


Los grupos son mutuamente excluyentes y totalmente incluyentes, por lo que cada mujer puede ser clasificada en un solo grupo y cada una tiene un grupo en el que ser incluida. Esto permite superar un problema que aparece frecuentemente cuando se clasifican las cesáreas de acuerdo con la indicación. Esta clasificación está basada en parámetros bien definidos y fáciles de controlar de cada mujer y su embarazo en lugar de la indicación de la cesárea, evitándose así mucha confusión, discrepancias y variabilidad inherente a la indicación de una cesárea. La Clasificación de Diez Grupos propuesta por Robson puede aplicarse internacionalmente y permite comparaciones en el tiempo de una misma unidad asistencial y entre distintas unidades (8-16).

El objetivo de este trabajo es realizar una auditoría en nuestro hospital, utilizando la clasificación estandarizada de Robson, para analizar los diferentes grupos de cesáreas y poder comparar las tasas obtenidas en los diferentes grupos con otros estudios tanto nacionales como internacionales, así como establecer las intervenciones posibles para reducir la tasa de cesáreas.

\section{MATERIAL Y MÉTODOS}

El trabajo se ha realizado en el Hospital comarcal la Inmaculada (Huércal-Overa/Almería), perteneciente al Servicio Andaluz de Salud (España), que atienda mayoritariamente población rural con una proporción de mujeres emigrantes del $26 \%$. Se atienden partos a partir de la semana 33-34, por debajo de esta edad gestacional son remitidos al Complejo hospitalario de Torrecardenas (Almería), por si los recién nacidos precisan UCI pediátrica.
Se han analizado 9.337 partos y 1.507 cesáreas lo que supone una frecuencia de cesáreas del $16,14 \%$, asistidas en un periodo de 8 años $\left(1^{\circ}\right.$ de enero de 2006 al 31 de diciembre de 2013). Los datos fueron extraídos retrospectivamente de la base de datos de partos.

Se realiza una auditoría retrospectiva, de las cesáreas en función de la clasificación de diez grupos establecida por Robson, para identificar grupos de mujeres susceptibles de obtener una reducción en el número de cesáreas.

La información necesaria de cada mujer embarazada para esta clasificación y análisis es: a) embarazo con feto único/múltiple; b) paridad; c) inicio del parto; d) edad gestacional; e) cesárea previa; y f) presentación fetal. Se analizaron también características maternas como la edad. La información fue analizada usando el programa estadístico IBM SPSS Statistics 20.0.

\section{RESULTADOS}

A continuación vamos desglosando todos los resultados, tal como se encuentran detallados en la Tabla II, en función de la clasificación por categorías de Robson.

La edad media materna al parto fue 28,51 años. Se observa un $71,2 \%$ de los partos de inicio espontáneo (6.649), $23,3 \%$ de inicio inducido (2.172) y un $5,5 \%$ cesáreas electivas (516). En cuanto a paridad: nulíparas el 49,4\% (4.616) y multíparas 50,6\% (4.721). Cesárea anterior en 789 casos (8,5\%). Número de embarazos con 1 feto (9.193), que representa el $98,5 \%$ y embarazos múltiples 144 partos $(1,5 \%)$.

Tabla II

APLICACIÓN DE LA CLASIFICACIÓN DE ROBSON EN NUESTRA POBLACIÓN

\begin{tabular}{cccccc}
\hline Grupo & Cesáreas/Parto & $\begin{array}{c}\text { Tamaño del } \\
\text { grupo (\%) }\end{array}$ & $\begin{array}{c}\text { \% de } \\
\text { cesáreas }\end{array}$ & $\begin{array}{c}\text { Contribución al } \\
\text { \% de cesáreas }\end{array}$ & $\begin{array}{c}\text { \% cesáreas/ } \\
\text { total de partos }\end{array}$ \\
\hline 1 & $263 / 2912$ & 31,2 & 9,0 & 17,4 & 2,8 \\
2 & $380 / 1268$ & 13,6 & 30,0 & 25,2 & 4,1 \\
3 & $66 / 3011$ & 32,2 & 2,19 & 4,4 & 0,7 \\
4 & $72 / 674$ & 7,2 & 10,7 & 4,8 & 0,8 \\
5 & $293 / 694$ & 7,4 & 42,2 & 19,4 & 3,1 \\
6 & $143 / 151$ & 1,6 & 94,7 & 9,5 & 1,5 \\
7 & $110 / 112$ & 1,3 & 98,2 & 7,3 & 1,2 \\
8 & $97 / 144$ & 1,5 & 67,4 & 6,4 & 1,0 \\
9 & $10 / 10$ & 0,1 & 100,0 & 0,7 & 0,1 \\
10 & $73 / 361$ & 3,9 & 20,2 & 4,9 & \\
\hline
\end{tabular}


Los grupos más numerosos son el grupo 1: nulíparas con un feto único en presentación cefálica, de 37 semanas o más de embarazo, que han iniciado el parto de forma espontánea: $(31,2 \%)$ y el grupo 3: multíparas sin cesárea previa, con un feto en presentación cefálica, de 37 o más de embarazo, que han iniciado el parto de forma espontánea $(32,2 \%)$.

El grupo menos numeroso, con $0,1 \%$, es el grupo 9: todas las mujeres con feto único en posición transversa u oblicua, incluyendo aquellas con cesárea previa, en el que se ha realizado un $100 \%$ de cesáreas.

Los grupos 6 y 7 (nulíparas y multíparas con fetos en presentación podálica incluyendo cesárea anterior) presentan una alta tasa de cesáreas. En el grupo 6 , se realizaron un $94,7 \%$ de cesáreas, hubo 8 partos vaginales en 7 años, lo cuales, acudieron a urgencias con parto en curso y no se realizó cesárea. En el grupo 7 se realizó un 98,2\% de cesáreas, hay dos casos de parto vaginal.

Respecto a los casos de embarazo múltiple (grupo 8), se han realizado un $67,4 \%$ de cesáreas.

Analizando los casos de cesárea anterior en el grupo 5 , se ha realizado un $42,2 \%$ de cesáreas.

En la contribución al porcentaje de cesáreas, en primer lugar está el grupo 2 (nulíparas con un feto único en presentación cefálica, de 37 semanas o más de embarazo, que han sido sometidas a inducción o cesárea antes del inicio del parto), que supone el $25,2 \%$ del total. En segundo lugar esta el grupo 5 (multíparas con al menos una cesárea previa, con un feto único en presentación cefálica, de 37 semanas o más de embarazo), con una contribución al porcentaje de cesáreas del 19,4\% y en tercer lugar el grupo 1 (nulíparas con un feto único en presentación cefálica, de 37 semanas o más de embarazo, que han iniciado el parto de forma espontánea), que representan el $17,4 \%$.

\section{DISCUSIÓN}

El aumento progresivo en la tasa de cesáreas nos lleva a analizar las diferentes situaciones y grupos de mujeres en los que ha aumentado la tasa de cesáreas para poder intervenir y frenar el aumento de las mismas, por eso la importancia de buscar sistemas de clasificación con los que realizar comparaciones (8-10).

La tasa de cesáreas en nuestro hospital es del $16,14 \%$, inferior a la publicada en España en el Ministerio de Sanidad que es del 24,9\% (aunque el dato es del 2011). Respecto a otros estudios realizados en España con clasificación de Robson con un porcentaje de cesáreas entre el (14-18,6\%) nos encontramos en cifras similares (11).

Un punto a tener en cuenta en la tasa de cesáreas es que en nuestro servicio no disponemos de alumnos de postgrado de forma rutinaria que puedan realizar indicaciones de cesáreas.

El tamaño de los grupos es similar al descrito en otras publicaciones siendo los grupos más frecuentes el grupo $1(11,14,16)$

El grupo con mayor contribución a la tasa de cesáreas es el grupo 2 que suponen el 25,2\%, similar a otros estudios (11). Para poder disminuir la sobreindicación de cesáreas en este grupo, se deberían intentar reducir tanto las inducciones como las cesáreas electivas. Las cesáreas electivas se deben presentar en sesión clínica y valorar cada caso específico, así como las cesáreas a demanda que suponen un reto al que nos debemos enfrentar con frecuencia. Las estimaciones de la prevalencia de partos por cesárea bajo demanda materna son del 1 a $18 \%$ de todos los partos por cesárea en todo el mundo (17). Por otro lado deberíamos tener claro las indicaciones de inducción para poder disminuir el número de cesáreas y guiarnos de protocolos actualizados en cada servicio de ginecología (6).

El grupo 5 supone el segundo conjunto en contribución al porcentaje de cesáreas con un 19,4\%. La tasa de cesárea primaria aumentada en las últimas décadas ha dado lugar a un gran número de mujeres sometidas a una o más cesáreas repetidas. En nuestro hospital se han realizado en este grupo un $42,2 \%$ cesáreas. El porcentaje de cesáreas descrito en este grupo en los artículos revisados de la literatura es muy variable desde el $21,3 \%$ al $84,2 \%$. $(9,11,14,15,16)$. Conviene tener en cuenta que hay evidencia científica de que las gestantes con una cesárea previa (transversal en segmento inferior), tiene un riesgo de ruptura uterina bajo $(0,7 \%)$ y una tasa de éxito de parto vaginal del $60-70 \%$ en estos casos (18).

El parto por cesárea es común en los embarazos de gemelos: más del $60 \%$ de los nacimientos de gemelos son por cesárea (19). Comparando las cesáreas en gestaciones múltiples con otros estudios observamos un porcentaje algo superior en nuestra población $(67,5 \%)$, no obstante la contribución al porcentaje de cesáreas es pequeña $6,4 \%$ por el tamaño reducido del grupo $(9,11,16)$. El tamaño del grupo de gemelos tiene tendencia a aumentar dado las técnicas de reproducción asistida, por lo que para poder disminuir el número de cesáreas en este grupo debemos tener en cuenta la posibilidad de parto vaginal cuando no existan contraindicaciones para el mismo. El consenso general es intentar un parto vaginal de gemelos con el primero en presentación cefálica independientemente de la presentación del segundo gemelo $(19,20)$.

Por consenso en nuestro hospital en todas las gestaciones con feto en podálica se realiza cesárea electiva por lo que los grupos 6 y 7 tienen un alto porcentaje de cesáreas, grupo 6 (94,7\%), grupo 7 $(98,2 \%)$ y suponen una contribución al porcentaje 
de cesáreas del $16,8 \%$. Hay una mayor mortalidad y morbilidad perinatal en fetos con presentación podálica que cefálica, debido principalmente a la prematuridad, malformaciones congénitas y asfixia perinatal o trauma en el parto, por lo que la finalización mediante cesárea electiva para la presentación podálica se ha sugerido como una forma de reducir los problemas perinatal asociada y en muchos países de Europa y de América se realiza cesárea electiva (21-24). En nuestro centro se ofrece la versión cefálica externa a aquellas gestantes con feto en presentación podálica en una gestación a término (37 semanas) y sin contraindicaciones para el procedimiento con el fin de reducir las presentaciones podálicas y las cesáreas por este tipo de presentación (25).

Respecto al grupo 10 (todas las mujeres con un feto único en presentación cefálica de menos de 37 semanas de embarazo, incluyendo aquellas con cesárea previa); en nuestro estudio el tamaño del grupo es menor que en otros estudios, así como el porcentaje de cesáreas $(8,11)$. Nuestro hospital no dispone de unidad de cuidados intensivos neonatales por lo que sólo se atiende partos con una edad gestacional igual o superior a 34 semanas. Entre 33 y 34 semanas se consensua la conducta, traslado o asistencia del parto en nuestro servicio, con el servicio de pediatría y con menos de 33 semanas se procede al traslado, si es posible, al hospital de referencia. Con este punto aclaramos que un grupo de riesgo como son los prematuros no son atendidos en nuestro hospital, lo que también podría influir en la tasa de cesáreas.

\section{CONCLUSIÓN}

El aumento de cesáreas que se esta produciendo en las últimas décadas hace necesario la realización de auditorías mediante un sistema de clasificación, como el de diez grupos descrito previamente, que nos permita comparar las tasa de cesáreas con otros hospitales y cuales son las medidas susceptibles de reducir el número de cesáreas. En nuestro caso el grupo que más contribuye al porcentaje de cesáreas es el número 2 que supone el $25,2 \%$ del total de cesáreas y aunque presenta un porcentaje similar a otros estudios es un grupo susceptible de cambio. Debemos analizar los casos de cesárea electiva en sesión clínica y revisar las indicaciones de inducción para poder disminuir el número de cesáreas.

\section{REFERENCIAS}

1. Aelvoet W, Windey F, Molenberghs G, Verstraelen $\mathrm{H}$, Van Reempts $\mathrm{P}$, Foidart JM. Screening for interhospital differences in cesarean section rates in lowrisk deliveries using administrative data: an initiative to improve the quality of care. BMC Health Serv Res 2008;8:3.
2. Anderson GM. Making sense of rising caesarean section rates. BMJ 2004;329:696-7.

3. Betran AP, Merialdi M, Lauer JA, Bing-shun W, Thomas $\mathrm{J}$, et al. Rates of caesarean section: analysis of global, regional and national estimates. Paediatr Perinat Epidemiol 2007;21:98-113.

4. World Health Organization. Appropiate technology for birth. Lancet 1985;2:436-7.

5. Dosa L. Caesarean section delivery, an increasingly popular option. Bull World Health Organ 2001;79(12):1173.

6. American College of Obstetricians and Gynecologists. ACOG committee opinion no. 559: Cesarean delivery on maternal request. Obstet Gynecol 2013;121:904.

7. Robson MS. Classification of Cesarean Sections. Fetal Maternal Med Rev 2001;12(1):23-39.

8. Chong C, Su LL, Biswas A. Changing trends of cesarean section births by the Robson. Ten Group Classification in a tertiary teaching hospital. Acta Obstet Gynecol Scand 2012;91(12):1422-7.

9. Robson MS. Can we reduce the caesarean section rate? Best Pract Res Clin Obstet Gynaecol 2001;15(1):179-94.

10. Robson MS, Scudamorel W, Walsh SM. Using the medical audit cycle to reduce cesarean section rates. Am J Obstet Gynecol 1996;174(1):199-205.

11. Cabeza Vengoechea PJ, Calvo Pérez A, Betrán AP, Mas Morey MM, Febles Borges MM, et al. (2010) Clasificación de cesáreas por Grupos de Robson en dos periodos comparativos en el Hospital de Manacor. Progres Obstet Ginecol 2010;53:385-90.

12. Abdel-Aleem $\mathrm{H}$, Shaaban OM, Hassanin Al, Ibraheem AA. Analysis of cesarean delivery at Assiut University Hospital using the Ten Group Classification System. Int J Gynecol Obstet 2013;123(2):119-23.

13. Florica M, Stephansson O, Nordstrom L. Indications associated with increased cesarean section rates in a Swedish hospital. Int J Gynaecol Obstet 2006;92:1815.

14. Vera C, Correa R, Neira J, Rioseco A, Poblete A. (2004). Utilidad de la evaluación de 10 grupos clínicos obstétricos para la reducción de la tasa de cesárea en un hospital docente. Rev Chil Obstet Ginecol 2004;69(3):219-26.

15. Costa ML, Cecatti JG, Souza JP, Milanez HM, Gülmezoglu MA. Research using a caesarean section classification system based on characteristics of the population as a way of monitoring obstetric practice. Reprod Health 2010;7:13.

16. McCarthy FP, Rigg L, Cady L, Cullinane F. A new way of looking at Caesarean section births. Aust N Z J Obstet Gynaecol 2007;47(4):316-20.

17. National Institutes of Health state-of-the-science conference statement: Cesarean delivery on maternal request March 27-29, 2006. Obstet Gynecol 2006;107:1386-97.

18. Guise J-M, Eden K, Emeis C, Denman MA, Marshall N, Fu R, Janik R, Nygren P, Walker M, McDonagh M. Vaginal birth after cesarean: new insights. Evidence report/technology Assessment No.191. (Prepared by the Oregon Health \& Science University Evidencebased Practice Center under Contract No. 290-200710057-I). AHRQ Publication No. 10-E003. Agency for Healthcare Research and Quality, Rockville, MD 2010. 
19. Steer P. Perinatal death in twins. BMJ 2007;334:5456.

20. Cruikshank DP. Intrapartum management of twin gestations. Obstet Gynecol 2007;109:1167-76.

21. Pritchard JA, MacDonald PC. Dystocia caused by abnormalities in presentation, position, or development of the fetus. In: Williams Obstetrics. Norwalk, CT: Appleton-Century-Crofts; 1980. pp 787-96.

22. Cheng M, Hannah M. Breech delivery at term: a critical review of the literature. Obstet Gynecol 1993;82:60518.
23. Danielian PJ, Wang J, Hall MH. Long-term outcome by method of delivery of fetuses in breech presentation at term: population based follow up. BMJ 1996;312:1451-3.

24. Hannah ME, Hannah WJ, Hewson SA, Hodnett ED, Saigal S, Willan AR. Planned caesarean section versus planned vaginal birth for breech presentation at term: a randomized multicentre trial. Lancet 2000;356:1375-83.

25. Kok M, Cnossen J, Gravendeel L, van der Post J, Opmeer B, Mol BW. Clinical factors to predict the outcome of external cephalic version: a metaanalysis. Am J Obstet Gynecol 2008;199 (6):630.e1-7. 